\title{
The Correlation Between the Treatment Efficacy and the Sympathetic Activity in Men With Lower Urinary Tract Symptoms
}

\author{
Hyun Ik Jang, Sung Gon Park, Kang Hee Shim, Jong Bo Choi, Jung Hwan Lee, Dae Sung Cho ${ }^{1}$ \\ Department of Urology, Ajou University School of Medicine, Suwon; \\ ${ }^{1}$ Department of Urology, Bundang Jesaeng Hospital, Seongnam, Korea
}

\begin{abstract}
Purpose: In this study, we examined the difference in the treatment efficacy depending on the sympathetic activity in men with lower urinary tract symptoms (LUTS).

Methods: In the current single-center, retrospective study, we evaluated a total of 66 male patients aged 40-70 years of age, presenting with LUTS, whose International Prostate Symptom Score (IPSS) exceeded 8 points. They had a past 3-month history of taking alfuzosin XL, and their heart rate variability (HRV) was measured before and after the treatment. In addition, we also recruited 39 healthy volunteers who visited a health promotion center for a regular medical check-up. They were aged between 40 and 70 years and had an IPSS of $<8$ points. We divided the patients with LUTS into two groups: the groups A and B, based on a low frequency/high frequency (LF/HF) ratio of 1.7, which was the mean value of the LF/HF ratio in the healthy volunteers. After a 3-month treatment with alfuzosin XL, we compared treatment outcomes, based on the IPSS and peak urine flow rate, between the two groups.

Results: A 3-month treatment with alfuzosin XL, comprising the measurement of the HRV, was performed for the 23 patients of the group A (23/38) and 17 of the group B (17/28). After a 3-month treatment with alfuzosin XL, total IPSS and IPSS questionnaire 2 and 5 were significantly lower in the group A as compared with the group B. But this was not seen in the group B. Furthermore, there were no significant differences in other parameters, such as maximal flow rate and IPSS storage subscore, between the two groups.

Conclusions: Our results indicate that the treatment efficacy was lower in patients with sympathetic hyperactivity as compared with those with sympathetic hypoactivity. Thus, our results will provide a basis for further studies to clarify causes of LUTS in a clinical setting.
\end{abstract}

Keywords: Autonomic nervous system; Heart rate; Lower urinary tract symptoms

\section{INTRODUCTION}

Lower urinary tract symptoms (LUTS) in men is a common symptom whose mechanism and etiology remain unclear [1]. It may be such a bothersome condition as to lead to the anxiety and even morbidity [2]. Moreover, due to the involvement of the prostate, LUTS shows different characteristics between men and women; voiding symptoms are more common in men than women. Idiopathic overactive bladder $(\mathrm{OAB})$ is common in both sexes. Most patients with idiopathic OAB suffer from frequency, urgency and nocturia. To date, many studies have mainly examined the involvement of muscarinic receptors and the nervous system innervating the bladder in the pathogenesis of $\mathrm{OAB}$. Moreover, it is widely known that the autonomic nervous system (ANS) is involved in the regulation of the micturition cycle. That is, there are several reports about the correlation between the activity of the ANS and the LUTS [3-6]. It is presumed that the central sympathetic overactivity might be associated with the
Corresponding author: Jong Bo Choi

Department of Urology, Ajou University School of Medicine, 206 World cup-ro, Yeongtong-gu, Suwon 443-721, Korea

Tel: +82-31-219-5273 / Fax: +82-31-219-5276 / E-mail: urochoi@ajou.ac.kr

Submitted: July 10, 2014 / Accepted after revision: September 15, 2014
This is an Open Access article distributed under the terms of the Creative Commons Attribution Non-Commercial License (http://creativecommons.org/licenses/by-nc/3.0/) which permits unrestricted non-commercial use, distribution, and reproduction in any medium, provided the original work is properly cited. 
LUTS in patients with benign prostatic hyperplasia (BPH); it is associated with metabolic syndrome in men [1,7].

The heart rate variability (HRV) can be used to assess the activity of the ANS. It is a quantitative, qualitative and noninvasive indicator of the systemic autonomic function. Autonomic dysfunction may be a causative factor for LUTS because the lower urinary tract is innervated and controlled by the ANS.

To date, few studies have been conducted to examine the correlation between the activity of the ANS and the LUTS in men, if any, and have suggested not only that it contributes to the pathophysology of the OAB but also that it may affect the treatment effects in male patients with LUTS.

Given the above background, we conducted this study to examine the difference in the treatment efficacy depending on the sympathetic activity in men with LUTS.

\section{MATERIALS AND METHODS}

In the current single-center, retrospective study, we evaluated a total of 66 male patients aged 40-70 years of age, presenting with LUTS, whose International Prostate Symptom Score (IPSS) exceeded 8 points. This study was initiated with approval of the Institutional Review Board of Ajou University College of Medicine (MED-MDB-12-273). They had a past 3-month history of taking alfuzosin XL, and their HRV was measured before and after the treatment. Thus, they were assigned to the patient group $(\mathrm{n}=66)$.

In addition, we also recruited 39 healthy volunteers who visited a health promotion center for a regular medical check-up. They were aged between 40 and 70 years and had an IPSS of $<8$ points. They had no underlying diseases that may affect the ANS, such as diabetes, cardiovascular disease, neurologic disturbance or any malignancies. In addition, they had no past history of surgery or pelvic trauma or that of taking medications that may affect the ANS. We excluded those who had a history of pelvic surgery or urinary retention, postvoiding residual urine of $>100$ and serum PSA of $>10 \mathrm{ng} / \mathrm{mL}$.

We informed the patients with a PSA level of $>4 \mathrm{ng} / \mathrm{mL}$ of the necessity to measure the PSA levels or to undergo prostate biopsy. We divided the patients with LUTS into two groups: the groups $\mathrm{A}$ and $\mathrm{B}$, based on a low frequency/high frequency (LF/ $\mathrm{HF}$ ) ratio of 1.7 , which was the mean value of the LF/HF ratio in the healthy volunteers (Table 1). After a 3-month treatment with alfuzosin XL, we compared treatment outcomes, based on the IPSS and peak urine flow rate, between the two groups.
Table 1. Clinical characteristics of 36 healthy volunteers

\begin{tabular}{lc}
\hline Characteristic & Value \\
\hline Age $(\mathrm{yr})$ & $56.17 \pm 6.63$ \\
Heart rate variability & \\
$\mathrm{LF}\left(\mathrm{ms}^{2}\right)$ & $210.99 \pm 264.05$ \\
$\mathrm{HF}\left(\mathrm{ms}^{2}\right)$ & $156.10 \pm 166.11$ \\
$\mathrm{LF} / \mathrm{HF}$ & $1.70 \pm 1.07$ \\
International Prostate Symptom Score & \\
Voiding subscore & $3.03 \pm 1.30$ \\
Storage subscore & $1.64 \pm 1.20$ \\
Total & $4.69 \pm 1.55$ \\
\hline
\end{tabular}

Values are presented as mean \pm standard deviation.

LF, low frequency; HF, high frequency.

\section{The Measurement of HRV}

The HRV was measured using the SA-3000p (Medicore, Seoul, Korea). At baseline, all the subjects received a bladder scan; the bladder contained at least $100 \mathrm{~mL}$ of urine. After a 30-minute resting, each subject underwent electrocardiographic signal recording for five minutes in a sitting position. With the subjects breathing normally, we calculated the mean heart rate and standard deviation of the N-N interval (SDNN) and the square root of the mean squared differences in successive N-N intervals (RMSSD) using time domain analysis. SDNN reflects all the cyclic components that are responsible for variability in the recording period and RMSSD represents the parasympathetic activity. But we did not compare the SDNN and RMSSD because it has been believed that a 5-minute HRV test is not sufficient. The parameters of the frequency domain analysis are total power, very low frequency, LF and LF/HF ratio. The HF peak of the spectrum $(0.15$ to $0.40 \mathrm{~Hz})$ represents parasympathetic activity, whereas the LF peak (0.04 to $0.15 \mathrm{~Hz}$ ) represents sympathetic activity. Thus, the LF/HF ratio represents the ANS balance (sympathetic to parasympathetic activity).

\section{Statistical Analysis}

Statistical analysis was performed using the IBM SPSS Statistics ver. 19.0 (IBM Co., Armonk, NY, USA). All data was expressed as mean \pm standard deviation. We also used the independent $t$ test to analyze differences in each parameter between the two groups. Furthermore, we performed the paired t-test to analyze the differences in each parameter between prior to and following the treatment. A P-value of $<0.05$ was considered statistically significant. 


\section{RESULTS}

\section{Baseline Characteristics of the Subjects}

The mean age of healthy volunteers was 56.2 years. Their mean LF/HF ratio was 1.7 (Table 1). Based on a cutoff value of LF/HF ratio of 1.7, $38(\mathrm{LF} / \mathrm{HF}$ ratio $<1.7)$ and 28 patients with LUTS $(\mathrm{LF} / \mathrm{HF}$ ratio $\geq 1.7)$ were assigned to the group $\mathrm{A}(\mathrm{n}=38)$ and the group $B(n=28)$, respectively. There were no significant dif-

Table 2. Patient characteristics and change of IPSS parameter and uroflowmetry in two subgroups of patients with lower urinary tract symptoms depending on the sympathetic activity

\begin{tabular}{lccc}
\hline Variable & $\begin{array}{c}\text { Group A } \\
(\mathrm{n}=38)\end{array}$ & $\begin{array}{c}\text { Group B } \\
(\mathrm{n}=28)\end{array}$ & P-value \\
\hline Age $(\mathrm{yr})$ & $59.11 \pm 9.92$ & $57.89 \pm 9.69$ & 0.62 \\
Volume of prostate & $31.74 \pm 10.29$ & $31.17 \pm 11.17$ & 0.84 \\
Baseline Qmax & $11.90 \pm 6.84$ & $11.92 \pm 7.10$ & 0.99 \\
Baseline IPSS total & $21.18 \pm 7.81$ & $18.00 \pm 7.86$ & 0.10 \\
Baseline IPSS voiding & $12.63 \pm 5.28$ & $10.89 \pm 5.29$ & 0.19 \\
Baseline IPSS storage & $7.50 \pm 4.24$ & $6.54 \pm 3.62$ & 0.34 \\
\hline
\end{tabular}

Values are presented as mean \pm standard deviation.

Group A, patients with $\mathrm{LF} / \mathrm{HF}<1.7$; group B, patients with $\mathrm{LF} / \mathrm{HF} 1.7$ and > 1.7; IPSS, International Prostate Symptom Score; LF, low frequency; HF, high frequency; Qmax, maximal flow rate. ferences in age, volume of the prostate, baseline maximal flow rate (Qmax) and IPSS total, IPSS voiding subscore (sum of IPSS questionnaire 1, 3, 5, and 6), and IPSS storage subscore (sum of IPSS questionnaire 2,4, and 7) at baseline (Table 2).

\section{Changes in IPSS and Qmax After a 3-Month Treatment With Alfuzosin XL}

A 3-month treatment with alfuzosin XL, comprising the measurement of the HRV, was performed for the 23 patients of the group A (23/38) and 17 of the group B (17/28). The results of IPSS score and Qmax are shown in Table 3. After a 3-month treatment with alfuzosin XL, total IPSS and IPSS questionnaire 2 and 5 were significantly lower in the group A as compared with the group B. But this was not seen in the group B. Furthermore, there were no significant differences in other parameters, such as Qmax and IPSS storage subscore, between the two groups (Table 3).

\section{DISCUSSION}

The overall prevalence of LUTS is high, and it increases with age, as does its severity [2]. It is commonly divided into the storage, voiding, and postmicturition symptoms. Histopathologically, it has been linked to the BPH. However, not all cases of LUTS are prostate related, and there is evidence demonstrat-

Table 3. Difference of the IPSS and Qmax after a 3-month treatment

\begin{tabular}{|c|c|c|c|c|c|c|}
\hline \multirow{2}{*}{ Parameter } & \multicolumn{3}{|c|}{ Group A $(n=23)$} & \multicolumn{3}{|c|}{ Group B $(n=17)$} \\
\hline & Baseline & After treatment & $\mathrm{P}$-value & Baseline & After treatment & P-value \\
\hline $\operatorname{Qmax}(\mathrm{mL} / \mathrm{sec})$ & $11.59 \pm 8.00$ & $11.70 \pm 6.01$ & 0.946 & $10.13 \pm 4.25$ & $12.91 \pm 7.85$ & 0.061 \\
\hline IPSS total & $20.93 \pm 9.75$ & $14.93 \pm 5.18$ & $0.006^{*}$ & $17.00 \pm 9.75$ & $17.11 \pm 7.11$ & 0.954 \\
\hline IPSS voiding & $12.86 \pm 5.22$ & $10.50 \pm 4.60$ & 0.077 & $9.78 \pm 5.78$ & $10.56 \pm 4.77$ & 0.211 \\
\hline IPSS storage & $6.79 \pm 4.98$ & $6.79 \pm 2.58$ & 1.000 & $6.78 \pm 4.47$ & $8.67 \pm 3.64$ & 0.170 \\
\hline IPSS No 1 & $3.73 \pm 1.74$ & $2.91 \pm 1.97$ & 0.381 & $1.50 \pm 1.87$ & $1.50 \pm 1.64$ & 1.000 \\
\hline IPSS No 2 & $2.55 \pm 2.07$ & $1.45 \pm 1.51$ & $0.038^{*}$ & $1.33 \pm 1.03$ & $2.50 \pm 1.38$ & 0.239 \\
\hline IPSS No 3 & $3.55 \pm 1.69$ & $2.10 \pm 1.70$ & 0.091 & $2.17 \pm 1.72$ & $0.83 \pm 0.75$ & 0.221 \\
\hline IPSS No 4 & $1.82 \pm 2.18$ & $0.82 \pm 0.98$ & 0.176 & $1.67 \pm 1.86$ & $1.50 \pm 1.76$ & 0.895 \\
\hline IPSS No 5 & $4.64 \pm 0.67$ & $2.73 \pm 1.68$ & $0.012^{*}$ & $4.33 \pm 0.82$ & $3.67 \pm 1.51$ & 0.444 \\
\hline IPSS No 6 & $2.45 \pm 2.02$ & $1.55 \pm 1.86$ & 0.346 & $2.67 \pm 1.03$ & $2.50 \pm 1.52$ & 0.833 \\
\hline IPSS No 7 & $2.64 \pm 1.80$ & $2.09 \pm 1.38$ & 0.441 & $1.67 \pm 0.82$ & $2.33 \pm 1.75$ & 0.484 \\
\hline IPSS No 8 (QoL) & $3.93 \pm 1.33$ & $3.07 \pm 1.35$ & 0.061 & $4.00 \pm 0.63$ & $3.50 \pm 0.55$ & 0.076 \\
\hline
\end{tabular}

Values are presented as mean \pm standard deviation.

Group A, patients with LF/HF < 1.7; group B, patients with LF/HF 1.7 and > 1.7; IPSS, International Prostate Symptom Score; Qmax, maximal flow rate; $\mathrm{LF}$, low frequency; HF, high frequency; QoL, quality of life.

${ }^{*} \mathrm{P}<0.05$ at the paired t-test. 
ing a direct link between the benign prostatic enlargement (BPE), bladder outlet obstruction (BOO) and LUTS [3]. The extended underlying pathophysiology leading to LUTS remains unclear, although it is increasingly recognized that the causes of LUTS extend well beyond BPE and BOO [8]. The multiple pathways leading to the onset and progression of LUTS not only complicate diagnosis but also limit the overall effectiveness and satisfaction of targeted symptom management strategies [9]. Therefore, treatment efficacy varies between individuals. Hyperactivity of the sympathetic nervous system may evoke LUTS, as shown in several studies about autonomic functions in male patients with LUTS. Previous epidemiological studies have suggested that there might be a relationship between the sympathetic overactivity and LUTS in male patients with BPH. Meigs et al. [10] noted that men with symptomatic BPH were also more likely to have characteristics associated with increased sympathetic activation, particularly in those who had heart disease, used $\beta$-blockers and lived a sedentary lifestyle.

The HRV is a simple and important tool for studying the autonomic control of the heart and autonomic dysfunction, and it represents one of the most promising markers [11,12]. We suggest the possibility of ANS imbalance and the use of HRV as a method to evaluate ANS tone. It depends on the effects of sympathetic and vagal activities on the sinus node, and its variability reflects spontaneous changes in autonomic activity [13]. It has been presumed that the HF and RMSSD are mainly responsive to changes in parasympathetic tone, whereas LF and SDNN are dually influenced by other physiologic inputs as well as cholinergic and adrenergic activities. Efferent vagal activity is a major contributor to the HF component, as is seen in clinical and experimental observations of autonomic maneuvers, such as electrical vagal stimulation, muscarinic receptor blockade and vagotomy [14-16]. Some authors reported that the interpretation of the LF component remains controversial [16-19], but others did that it is a marker of the sympathetic modulation when it is particularly expressed in normalized units [14].

It has been previously shown that there is an increase in the sympathetic tone in patients with LUTS and a greater increase in it in those with voiding symptom. According to Oh et al. [20], however, the severity of storage symptom was greater in patients with sympathetic hypoactivity, thus presenting with frequency, as compared with those with sympathetic hyperactivity.

We conducted the current study to examine the difference in the treatment efficacy between patients with LUTS with sympathetic hyperactivity and those with sympathetic hypoactivity.
The degree of the treatment efficacy is relatively lower in male patients with LUTS who had sympathetic hyperactivity as compared with those with sympathetic hypoactivity. Alfuzosin is a selective $\beta$-blocker but it does not have selectivity for the $\alpha 1$ receptor subtypes. This explains the reason to ignore the difference of sympathetic activity. It can therefore be inferred that there would be different outcomes if there are any chances that ala-selective blockers, such as tamsulosin or silodosin, are used. This deserves further studies.

There are several limitations of the current study are as follows:

(1) We enrolled a small number of patients in the current study. It was a pilot study to determine the role of autonomic activity in the treatment of patients with LUTS. This deserves further large-scale studies.

(2) The LUTS are not specific but subject to change depending on the factors that are not associated with prostatic conditions such as diet, fluid intake, alcohol intake, anticholinergic effects of commonly used over-the-counter medications, smoking and even mood or physical activity [21-23]. In the current study, we attempted to control such effects by encouraging the patients not to consume anything that may affect the activity of the ANS prior to the measurement of the HRV.

(3) We enrolled relatively younger subjects as compared with a general group of patients with LUTS. The age difference might have been due to exclusion criteria. We excluded men with underlying diseases that may affect the activity of the ANS, such as diabetes and hypertension. Such diseases commonly show a lower prevalence in younger men who commonly take less medications that may affect the activity of the ANS. Many patients with LUTS who do not respond to current medications or surgical treatments have a poor quality of life. Our results suggest that ANS disturbances, especially sympathetic hyperactivity, may be involved in the progression of diseases in men with LUTS.

In conclusion, our results indicate that the treatment efficacy was lower in patients with sympathetic hyperactivity as compared with those with sympathetic hypoactivity. Thus, our results will provide a basis for further studies to clarify causes of LUTS in a clinical setting.

\section{CONFLICT OF INTEREST}

No potential conflict of interest relevant to this article was reported. 


\section{ACKNOWLEDGEMENTS}

This study was supported by a grant from Ajou University Hospital.

\section{REFERENCES}

1. McVary KT, Rademaker A, Lloyd GL, Gann P. Autonomic nervous system overactivity in men with lower urinary tract symptoms secondary to benign prostatic hyperplasia. J Urol 2005;174(4 Pt 1): 1327-433.

2. Ushijima S, Ukimura O, Okihara K, Mizutani Y, Kawauchi A, Miki T. Visual analog scale questionnaire to assess quality of life specific to each symptom of the International Prostate Symptom Score. J Urol 2006;176:665-71.

3. Choi JB, Lee JG, Kim YS. Characteristics of autonomic nervous system activity in men with lower urinary tract symptoms (LUTS): analysis of heart rate variability in men with LUTS. Urology 2010; 75:138-42.

4. Hubeaux K, Deffieux X, Raibaut P, Le Breton F, Jousse M, Amarenco G. Evidence for autonomic nervous system dysfunction in females with idiopathic overactive bladder syndrome. Neurourol Urodyn 2011;30:1467-72.

5. Liao WC, Jaw FS. A noninvasive evaluation of autonomic nervous system dysfunction in women with an overactive bladder. Int J Gynaecol Obstet 2010;110:12-7.

6. Ben-Dror I, Weissman A, Leurer MK, Eldor-Itskovitz J, Lowenstein L. Alterations of heart rate variability in women with overactive bladder syndrome. Int Urogynecol J 2012;23:1081-6.

7. Kirby MG, Wagg A, Cardozo L, Chapple C, Castro-Diaz D, de Ridder $\mathrm{D}$, et al. Overactive bladder: is there a link to the metabolic syndrome in men? Neurourol Urodyn 2010;29:1360-4.

8. Bushman W. Etiology, epidemiology, and natural history of benign prostatic hyperplasia. Urol Clin North Am 2009;36:403-15.

9. McConnell JD, Roehrborn CG, Bautista OM, Andriole GL Jr, Dixon CM, Kusek JW, et al. The long-term effect of doxazosin, finasteride, and combination therapy on the clinical progression of benign prostatic hyperplasia. N Engl J Med 2003;349:2387-98.

10. Meigs JB, Mohr B, Barry MJ, Collins MM, McKinlay JB. Risk factors for clinical benign prostatic hyperplasia in a community-based population of healthy aging men. J Clin Epidemiol 2001;54:935-44.

11. Task Force of the European Society of Cardiology and the North
American Society of Pacing and Electrophysiology. Heart rate variability. Standards of measurement, physiological interpretation, and clinical use. Eur Heart J 1996;17:354-81.

12. Silvetti MS, Drago F, Ragonese P. Heart rate variability in healthy children and adolescents is partially related to age and gender. Int J Cardiol 2001;81:169-74.

13. Choi JB, Kim YB, Kim BT, Kim YS. Analysis of heart rate variability in female patients with overactive bladder. Urology 2005;65: 1109-12.

14. Akselrod S, Gordon D, Ubel FA, Shannon DC, Berger AC, Cohen RJ. Power spectrum analysis of heart rate fluctuation: a quantitative probe of beat-to-beat cardiovascular control. Science 1981;213:220-2.

15. Pomeranz B, Macaulay RJ, Caudill MA, Kutz I, Adam D, Gordon $\mathrm{D}$, et al. Assessment of autonomic function in humans by heart rate spectral analysis. Am J Physiol 1985;248(1 Pt 2):H151-3.

16. Malliani A, Pagani M, Lombardi F, Cerutti S. Cardiovascular neural regulation explored in the frequency domain. Circulation 1991; 84:482-92.

17. Kamath MV, Fallen EL. Power spectral analysis of heart rate variability: a noninvasive signature of cardiac autonomic function. Crit Rev Biomed Eng 1993;21:245-311.

18. Rimoldi O, Pierini S, Ferrari A, Cerutti S, Pagani M, Malliani A. Analysis of short-term oscillations of R-R and arterial pressure in conscious dogs. Am J Physiol 1990;258(4 Pt 2):H967-76.

19. Montano N, Ruscone TG, Porta A, Lombardi F, Pagani M, Malliani A. Power spectrum analysis of heart rate variability to assess the changes in sympathovagal balance during graded orthostatic tilt. Circulation 1994;90:1826-31.

20. Oh DG, Cho DS, Yun IS, Lee KB, Choi JB, Lee JH. The difference of lower urinary tract symptoms between sympathetic hyperactive and hypoactive men. Int Neurourol J 2013;17:30-3.

21. Boyle P, Robertson C, Mazzetta C, Keech M, Hobbs FD, Fourcade R, et al. The prevalence of lower urinary tract symptoms in men and women in four centres. The UrEpik study. BJU Int 2003;92:409-14.

22. Fowke JH, Phillips S, Koyama T, Byerly S, Concepcion R, Motley SS, et al. Association between physical activity, lower urinary tract symptoms (LUTS) and prostate volume. BJU Int 2013;111:122-8.

23. Harte CB, Liverant GI, Sloan DM, Kamholz BW, Rosebrock LE, Fava $\mathrm{M}$, et al. Association between smoking and heart rate variability among individuals with depression. Ann Behav Med 2013;46: 73-80. 